\title{
Temperature dependence of the $x$-ray photoemission line shape and of the hopping rate in a marginal Fermi liquid
}

\author{
H J M van Bemmel and $\mathrm{W}$ van Saarloos \\ Institute Lorentz, University of Leiden, PO Box 9506, 2300 RA Leiden, The Netherlands
}

Received 10 November 1992

\begin{abstract}
We study the spectral properties of a localized particle (a deep core level, or a heavy particle hopping in a solid), coupled to conduction electrons that are described by a marginal Fermi liquid hypothesis. Our main result is that, in this model, the core level line shape in an $x$-ray photoemission experiment shifts with temperature. The spectrum we find is consistent with the decrease of the hopping rate with decreasing temperature obtained by Zhang $e t$ al. In the presence of a distribution of energy levels for the hopping particle, the temperature dependence of the hopping rate is found to be less pronounced. The observability of both the shift in the $x$-ray photoemission spectrum and the decrease of the hopping rate depends on the strength of the interaction. Our rough estimate of the value of the interaction parameter indicates that the effect might be observable in $\mathrm{x}$-ray photoemission experiments.
\end{abstract}

\section{Introduction}

As is well known, the normal-state properties of high-temperature superconductors differ in many ways from those of classical superconductors. To describe these differences empirically, Varma et al $[1,2]$ recently introduced the marginal Fermi liquid (MFL) hypothesis, a phenomenological ansatz for the electronic polarizability. From this ansatz, they can reproduce a large number of the characteristic features of the high-temperature superconductors. At present, though, a microscopic basis for this MFL hypothesis is lacking, nor is it clear to what extent the ansatz can really be self-consistent.

In the absence of a theory, it is important to try to assess the range of validity of the MFL hypothesis as much as possible, by comparing its predictions with experiments. Recently, Zhang et al [3] pointed out that the hopping of a localized particle that interacts with an MFL differs significantly from that of a particle interacting with an ordinary Fermi liquid (FL): in an MFL, the hopping rate $\nu$ goes down as the temperature is lowered, whereas in an FL it goes up with decreasing temperature. This is due to the vanishing of the quasi-particle spectral weight at the Fermi surface in the MFL ansatz, or, equivalently, due to the logarithmic divergence of the effective mass as $T \rightarrow 0$ in an MFL. In a metal, the probability of a particle hopping to a neighbouring site is lowered because of the interaction with the conduction electrons. The overlap of the many-electron wave functions with the particle at different sites is smaller than unity-in fact it tends to zero for a large system, no matter how small the potential is (Anderson's orthogonality catastrophe [7]). In a normal metal, this effect gives rise to a power law divergence of the hopping rate $\nu$ with temperature. 
However, according to the results of Zhang et al [3], for an MFL $\nu$ strongly decreases at low temperatures.

Another problem which is connected with the orthogonality catastrophe is the $\mathrm{x}$-ray edge effect. In $\mathrm{x}$-ray photoemission (we use the common abbreviation XPS, for $x$-ray photoelectron spectroscopy), the core electron that is shot out of the material with the $x$-rays leaves a positive hole. The change in the potential is similar to that in the hopping problem, and in this case the photoemission line shape is determined by the overlap of the many-electron wave functions with and without the hole. The resulting theoretical XPS line shape is very asymmetrical and falls off on one side as a power law of the energy of the outcoming electron. In practice, lifetime broadening reduces the asymmetry of the line shape [10].

Given the similarity of the hopping problem and the $x$-ray problem, the results of Zhang et al [3] lead one to suspect that interesting differences exist in the XPS line shape depending on whether the core hole interacts with an FL or with an MFL. It is the purpose of this paper to investigate whether this is indeed the case, and to assess the feasibility of measuring these differences experimentally. As we shall see, the power law behaviour in the XPS line shape is suppressed in the case of an MFL, while the position of the peak acquires an interesting temperature dependence which is not found for an FL. Whether this temperature dependence can be seen experimentally, however, depends sensitively on the parameters, in particular the strength of the interaction: our results indicate that for small interaction strength, the anomalous temperature dependence of the hopping rate predicted by Zhang et al [3] should be measurable but the temperature dependence of the XPS peak will be difficult to observe; for larger interaction parameters, however, the situation is reversed: the latter effect is then more likely to be measurable than the former.

In XPS, the electrons come out of the material with different kinetic energies, large enough that there are no final state interactions with the material. The $\mathrm{x}$-ray energy is used to supply the binding energy of the core electron as well as the energy necessary for the creation of different amounts of electron-hole pairs, needed to overcome the orthogonality of the many-electron wave functions. One measures the distribution of outcoming kinetic energy, which is determined by the spectral function of the core hole, $A_{b}(\omega)$. In the case of hopping, there is no freedom for the final energy of the particle, as it has to match the energy of the level at the neighbouring site. If the energies at different sites are the same, we have $\nu \propto A_{\mathrm{b}}(0)$. If there is a distribution of levels, however, we have to average $A_{\mathrm{k}}(\omega)$ over a certain energy (or frequency) interval. We shall see that such an averaging can affect the results for the hopping rather dramatically in the case of an MFL, since the decrease of $\nu$ with decreasing temperature turns out to be related to a slight temperature dependent shift in the $x$-ray spectrum, not to a decrease of the maximum itself. As a result, upon averaging, the tendency of the hopping rate $\nu$ to decrease with temperature is reduced. This sensitivity of the result to inhomogeneities could be relevant in the high-temperature superconductors.

It is important that both the hopping problem and the photoemission problem are determined by orthogonality alone, whereas other analogous local problems involve a competing singular effect. For example, in the case of $x$-ray absorption, the core electron does not leave the material; instead, there is a tendency to form a bound state with the core hole (the exciton effect, see e.g. Mahan [8]). However, in the absence of a microscopic theory for the $\mathrm{MFI}$, the absorption cannot at present be studied with the MFL hypothesis. That non-Fermi liquid effects can also be important 
for such absorption spectra has very recently been demonstrated for one-dimensional Luttinger liquids $[16,17]$. In XPS, however, final-state interactions are absent, and the effects of orthogonality can be analysed completely [10] in terms of the expression for the electronic polarizability provided by the MFL hypothesis alone.

It is interesting to note that the important differences between the $\mathrm{FL}$ and the MFL spectra result from the differences in the electronic polarizability of the two cases. In principle, therefore, an experimental investigation of the spectrum allows one to test the essential part of the MFL hypothesis that the anomalous normal-state properties are associated with an anomalous electronic polarizability. Other theories of the normal-state behaviour, e.g. for the linear dependence of the resistivity with temperature, do not necessarily led to the same result.

In the next sections, we shall first calculate the theoretical line shapes for the MFL at different temperatures, and compare them to the ones for an FL. After this, we will give a discussion of the observability of the predicted effects.

\section{Formulation of the problem}

There exist various formulations of both the hopping problem $[4-6,13]$ and of manybody effects in $\mathrm{x}$-ray spectroscopy $[10,14,15]$. We choose a formulation which involves the electronic polarizability in the frequency domain.

The model that we consider is that of a particle (the hopping particle or a corelevel electron) in a localized state coupled to the conduction electrons through an interaction potential $V$. The properties of the conduction electrons are assumed to be described by the MFL ansatz for the electronic polarizability $P(q, \omega)$. For both problems, the interaction is of the form $\sum_{q k} V(q) a_{k+q}^{\dagger} a_{k}$, where $a_{k}$ and $a_{k}^{\dagger}$ are creation and annihilation operators for the conduction electrons. For the hopping problem $V(q)$ is the Fourier transform of the difference in the potential that the conduction electrons feel when the particle hops to a neighbouring site with the same energy, while for the $\mathrm{x}$-ray problem $V(\boldsymbol{q})$ is the effective potential that the conduction electrons experience when the core level is unoccupied [8].

For the hopping problem, the hopping rate $\nu$ between two levels of the same energy is given by [3]

$$
\nu=4 \Delta^{2} \operatorname{Re} \int_{0}^{\infty} \mathrm{d} t \rho(t) .
$$

Here $\Delta$ is the tunnelling matrix element without any interaction and $\rho(t)=$ $\left\langle\mathrm{e}^{\mathrm{i} \mathcal{H}\left(\boldsymbol{R}_{2}\right) \mathrm{t}} \mathrm{e}^{-\mathrm{i} \mathcal{H}\left(\boldsymbol{R}_{1}\right) \eta}\right\rangle$, where $\mathcal{H}\left(\boldsymbol{R}_{1}\right)$ is the interaction Hamiltonian with the hopping particle at site $\boldsymbol{R}_{1}$ and $\mathcal{H}\left(\boldsymbol{R}_{2}\right)$ the Hamiltonian when the particle is at site $\boldsymbol{R}_{2}$. As usual, we take $\hbar=1$.

In the case of photoemission, the relevant quantity is $A_{\mathrm{b}}(\omega)$, the spectral function of the core hole. This quantity directly determines the line shape, and is given by

$$
A_{\mathrm{h}}(\omega)=2 \operatorname{Re} \int_{0}^{\infty} \mathrm{d} t \mathrm{e}^{\mathrm{i} \omega t} \rho(t)
$$

where in this case $\rho(t)=\left\langle\mathrm{e}^{\mathrm{i} \mathcal{H}_{\mathrm{s}} \mathrm{e}} \mathrm{e}^{-\mathrm{i} \mathcal{H}_{\mathrm{c}} 山}\right\rangle$, with $\mathcal{H}_{\mathrm{g}}$ the ground-state Hamiltonian (with the core level occupied) and $\mathcal{H}_{\mathrm{f}}$ the Hamitonian with the core hole present [8]. The 
core hole Green function equals $-\mathrm{i} \Theta(t) \rho(t)$. In equation (2), we measure the energy $\omega$ relative to the energy in the absence of interaction with the conduction electrons; without these interactions, the spectral density is just a delta function at $\boldsymbol{\omega}=\mathbf{0}$.

The function $\rho(t)$ is normally analysed by writing $\rho(t)=\mathrm{e}^{F(t)}$ and calculating $F(t)$ with the aid of a linked cluster expansion, $F(t)=\sum_{l} F_{l}(t)$. The general expression for $F_{2}(t)$ is [4]

$$
F_{2}(t)=\frac{1}{\Omega \pi} \int_{-\infty}^{\infty} \frac{\mathrm{d} \omega}{\omega^{2}} \frac{1-\mathrm{e}^{-\mathrm{i} \omega t}}{1-\mathrm{e}^{-\beta \omega}} \sum_{\boldsymbol{q}}\left(\frac{V\left(q_{r}\right)}{\epsilon\left(q_{r}\right)}\right)^{2} \operatorname{Im} P(q, \omega)
$$

where $\Omega$ is the volume of the system $\operatorname{Im} P(q, \omega)$ is the imaginary part of the electronic polarizability; $\epsilon(q)$ is the $\omega \rightarrow 0$ limit of the dielectric function $\epsilon(q, \omega)$, which is the relevant limit for this problem.

For the free electron gas, higher-order terms $F_{l}$ with $l>2$ are known [14] to be quantitatively important, but they do not change the qualitative behaviour. We will ignore such higher-order terms here since they cannot be analysed without a microscopic theory for the MFL.

Equation (3) provides a suitable starting point for comparing the behaviour of an FL with that of the MFL hypothesis, since the latter is formulated directly in terms of the polarizability $P$,

$$
\operatorname{Im} P^{\mathrm{MFL}}(q, \omega)=-\frac{2 N_{\mathrm{F}}}{\pi} \frac{\tanh (\omega / 2 T)}{1+\left(\omega / \omega_{\mathrm{c}}\right)^{2}}
$$

where $N_{\mathrm{F}}$ is the density of states at the Fermi level, and where $\omega_{\mathrm{c}}$ is a frequency cut-off which is of the order of a few hundred meV (a few thousand Kelvin; we take both $h$ and $k_{\mathrm{B}}$ equal to 1 ).

It has already been noted by Zhang et al [3] that there are conceptual difficulties with this ansatz: it does not satisfy the usual $f$-sum rule [8]. This problem cannot be remedied by including a $q$-dependent function on the right-hand side. These conceptual difficulties have, however, also practical implications, in that they make it difficult to estimate the magnitude of the $\mathrm{x}$-ray edge effect or of the temperature dependence of the hopping. As we shall see, when equation (4) is taken at face value, one is led to expect a measurable temperature dependence of the XPS line shape (in particular a temperature dependent frequency shift), and a strongly suppressed hopping rate [19].

To see this, let us compare with the case of an FL. In this case, we have to a good approximation [8]

$$
\operatorname{Im} P^{\mathrm{FL}}(q, \omega)=-\omega\left(2 \pi^{2} N_{\mathrm{F}}^{2} / q k_{\mathrm{F}}^{2}\right) \Theta\left(2 k_{\mathrm{F}}-q\right)
$$

so that, upon introducing a cut-off $\omega_{\mathrm{c}}$ which is of the order of $\epsilon_{F}$

$$
F_{2}^{\mathrm{FL}}(t)=-K^{\mathrm{FL}} \int_{-\omega_{\varepsilon}}^{\omega_{\epsilon}} \frac{\mathrm{d} \omega}{\omega} \frac{1-\mathrm{e}^{-\mathrm{i} \omega t}}{1-\mathrm{e}^{-\beta \omega}}
$$

where

$$
K^{\mathrm{FL}}=\frac{1}{\Omega \pi} \frac{2 \pi^{3} N_{\mathrm{F}}^{2}}{k_{\mathrm{F}}^{2}} \sum_{q}\left(\frac{V(q)}{\epsilon(q)}\right)^{2} \frac{1}{q} \Theta\left(2 k_{\mathrm{F}}-q\right)=\mathcal{O}\left(N_{\mathrm{F}} V\right)^{2}
$$


Here $V$ is the order of magnitude of $V(q)$. For an FL, the dimensionless number $K^{F L}$ is directly related to the phase shift induced by the potential; a typical value for $K^{F L}$ that we get from the experiments is $0.1[8,11]$.

Let us now compare these results with those for an MFL. First of all, experiments show that the high-temperature superconductors show strong Raman scattering. This indicates at least that equation (4) is indeed of the right order of magnitude in the regime relevant for Raman scattering (assuming that the coupling to light is not drastically different from that of other materials). At typical frequency shifts $\omega$ of the order of hundred $\mathrm{cm}^{-1}$, i.e. of the order of $10^{-1} E_{\mathrm{F}}$ or less, and wavenumbers $q \approx 10^{-3} k_{\mathrm{F}}, \operatorname{Im} P^{\mathrm{FL}}$ is of the order of $N_{\mathrm{F}}$ or somewhat larger. Expression (4) for $\operatorname{Im} P^{\mathrm{MFL}}$ also gives a result of order $N_{\mathrm{F}}$ for $\omega \geqslant T$.

Using equation (4) in equation (3) gives

$$
F_{2}^{\mathrm{MFL}}(t)=-K^{\mathrm{MFL}} \int_{-\infty}^{\infty} \frac{\mathrm{d} \omega}{\omega^{2}} \frac{1-\mathrm{e}^{-\mathrm{i} \omega t}}{1-\mathrm{e}^{-\beta \omega}} \frac{\tanh (\beta \omega / 2)}{1+\left(\omega / \omega_{\mathrm{c}}\right)^{2}}
$$

with

$$
K^{\mathrm{MFL}}=\frac{2 N_{\mathrm{F}}}{\Omega \pi^{2}} \sum_{q}\left(\frac{V(q)}{\epsilon(q)}\right)^{2}=\mathcal{O}\left(N_{\mathrm{F}} k_{\mathrm{F}}^{3} V^{2}\right)
$$

Let us assume that the potential $V$ is of the same order in the high- $T_{c}$ materials as it is in normal metals. Then we get [20]

$$
K^{\mathrm{MFL}}=\mathcal{O}\left(N_{\mathrm{F}}^{\mathrm{MFL}} k_{\mathrm{F}}^{3} /\left(N_{\mathrm{F}}^{\mathrm{FL}}\right)^{2}\right) K^{\mathrm{FL}}=\mathcal{O}\left(0.1 E_{\mathrm{F}}\right) \approx 1000 \mathrm{~K}
$$

We stress that this is a very rough estimate, as it is based on various rather crude approximations; the interaction parameter will depend strongly on the closeness of the relevant site to the copper oxide planes in a high- $T_{\mathrm{c}}$ superconductor, and can be different for the hopping and the $\mathrm{x}$-ray problem. In particular, it seems likely to us that equation (4) overestimates the polarizability considerably for short wavelength (note that $\operatorname{Im} P^{\mathrm{FL}}$ decreases as $q^{-1}$ ) ; thus we think our expression overestimates $K^{\mathrm{MFL}}$. Moreover, any effects of anisotropy, which should be quite important for the cuprates, have been ignored. We finally note that Zhang et al [3] used $K^{-\mathrm{MFL}}=15 \mathrm{~K}$, without justifying this particular choice.

\section{Comparison of $F_{2}^{\mathrm{FL}}$ and $F_{2}^{\mathrm{MFL}}$}

The integral in equation (6) for an FL with a sharp cut-off can be done [9] but for our numerical studies we choose to evaluate this integral with a smooth cut-off, of the same form as the high-frequency cut-off in the MFL expression.

Consider $F_{2}^{\mathrm{MFL}}$, given by equation (8). The contour can be closed in the lower half plane. There are poles in $0,-i \omega_{c}$ and $-2 n \pi T i, n=1,2, \ldots$. The result for the principal part of the integral is that the real part has a simple expression:

$$
\operatorname{Re} F_{2}^{\mathrm{MFL}}(t)=-K^{\mathrm{MFL}}\left(\frac{\pi t}{2}-\frac{\pi}{2 \omega_{\mathrm{c}}}\left(1-\mathrm{e}^{-\omega_{\mathrm{c}} t}\right)\right)
$$




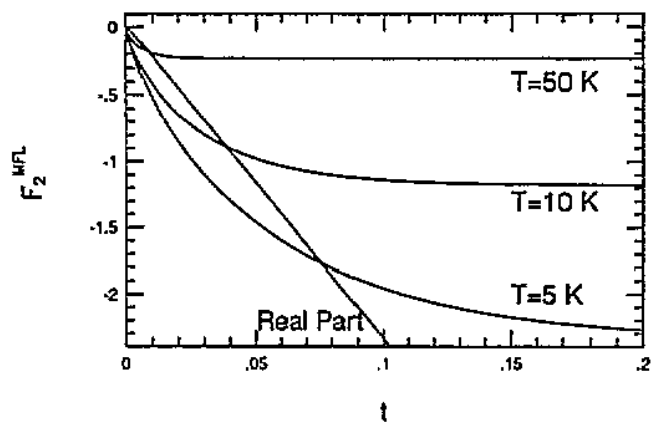

Figure 1. Behaviour of $\operatorname{Im} F_{2}^{\mathrm{MFL}}$ and $\operatorname{Re} F_{2}^{\mathrm{MFL}}$ as a function of time, with $K^{\mathrm{MFL}}=15 \mathrm{~K}$ and $\omega_{\mathrm{c}}=1500 \mathrm{~K}$. The real part of $F_{2}^{\mathrm{MFL}}$ is independent of temperature.

and that the imaginary part contains an infinite sum of contributions of the poles $-2 n \pi T i$. These become small for large $n$, and we can sum them numerically until we obtain the desired precision.

The above result agrees with that of Zhang et al for small $t$, but their expression is wrong for large $t$. In particular, their logarithmic correction is absent; this does not affect the results significantly, however. Note that, as Zhang et al already stressed, $\operatorname{Re} F_{2}^{\mathrm{MFL}}$ is temperature independent and decreases linearly for large times. In an $\mathrm{FL}$, on the other hand, $\operatorname{Re} F_{2}^{\mathrm{FL}}$ has strong temperature dependence, and at $T=0$ it decreases logarithmically for long times; for finite temperature, the asymptotic behaviour is $-K^{-F L} \pi t T$.

To obtain the asymptotic behaviour of $\operatorname{Im} F_{2}^{\mathrm{MFL}}$, we note that by using the fact that $\left(1-\mathrm{e}^{-\beta \omega}\right)^{-1}=1-\left(\mathrm{e}^{\beta \omega}-1\right)^{-1}$, we can write

$$
\operatorname{Im} F_{2}^{\mathrm{MFL}}(t)=-K^{\mathrm{MFL}} \int_{0}^{\infty} \frac{\mathrm{d} \omega}{\omega^{2}} \frac{\sin \omega t \tanh (\beta \omega / 2)}{1+\left(\omega / \omega_{\mathrm{c}}\right)^{2}} .
$$

For $t \rightarrow \infty, \sin \omega t / \omega \rightarrow \pi \delta(\omega)$, and we find

$$
\operatorname{Im} F_{2}^{\mathrm{MFL}}(t \rightarrow \infty)=-\pi \beta K^{\mathrm{MFL}} / 4 \text {. }
$$

By differentiating equation (12) with respect to $t$, we get

$$
\left.\frac{\mathrm{d} \operatorname{Im} F_{2}^{\mathrm{MFL}}(t)}{\mathrm{d} t}\right|_{t=0}=-K^{\mathrm{MFL}} \int_{0}^{\infty} \frac{\mathrm{d} \omega}{\omega} \frac{\tanh (\beta \omega / 2)}{1+\left(\omega / \omega_{\mathrm{c}}\right)^{2}}
$$

For $\beta \omega_{c} \gg 1$, the integrand behaves as $\omega^{-1}$ over a large part of the $\omega$-interval, and this results in a logarithmic temperature dependence, $\ln \beta \omega_{c^{*}}$. In particular, if we replace the smooth cut-off by a sharp cut-off at $\omega_{c}$, the integral in equation (14) is of the form well known in the BCS theory, and we get

$$
\left.\frac{\mathrm{d} \operatorname{Im} F_{2}^{\mathrm{MFL}}(t)}{\mathrm{d} t}\right|_{t=0}=-K^{\mathrm{MFL}} \ln 1.13 \beta \omega_{\mathrm{c}} .
$$

With a smooth cut-off, the numerical constant 1.13 is replaced by another constant of order unity.

The behaviour of $\operatorname{Im} F_{2}^{\mathrm{MFL}}$ as a function of time is illustrated in figure 1 for several temperatures and $K^{\mathrm{MFL}}=15 \mathrm{~K}$. We see that the asymptotic value, equation (13), is approached smoothly, the approach being slower at lower temperatures. This is a result of the fact that the $t=\infty$ value diverges as $T^{-1}$, while the initial slope diverges only logarithmically. 


\section{Consequences for $A_{\mathrm{h}}(\omega)$}

Before presenting the results for the MFL case, let us recall the essential features of the spectral density in the case of an ordinary FL, obtained numerically from equation (2) in the approximation $\rho=\mathrm{e}^{F_{2}^{\mathrm{FL}}(t)}$. At temperature $T=0$, the asymptotic $\ln t$ dependence of $\operatorname{Re} F_{2}^{\mathrm{FL}}$ gives rise to the well known $\omega^{-\left(1-K^{\mathrm{PL}}\right)}$ divergence of the spectrum for positive $\omega$, while $A_{b}(\omega)=0$ for $\omega<0$. As illustrated in figure 2 , for finite temperatures the line shape starts to broaden and to become less asymmetric (since $\omega$ is given in units of 'temperature', figure 2 illustrates that the width of the spectrum for $\omega<0$ is of order $T$ ). At the same time, there is a small shift in the position of the peak with increasing temperature, and the peak height drops. Since the shift of the peak is small, and since the small- $\omega$ behaviour is determined by the asymptotic behaviour of $F_{2}^{\mathrm{FL}}$, we can use the results $\operatorname{Re} F_{2}^{\mathrm{FL}} \approx-K^{\mathrm{FL}} \pi / 2$ and $\operatorname{Im} F_{2}^{\mathrm{FL}} \approx-K^{\mathrm{FL}} \pi t T$ for $t \rightarrow \infty$ of the previous section to obtain the following result for $A_{b}(\omega)$, valid for small $\omega$ and $T$ :

$$
A_{\mathrm{h}}^{\mathrm{FL}}(\omega) \approx \frac{K^{\mathrm{FL}} \pi T \cos \left(K^{\mathrm{FL}} \pi / 2\right)+\omega \sin \left(K^{\mathrm{FL}} \pi / 2\right)}{\left(K^{\mathrm{FL}} \pi T\right)^{2}+\omega^{2}} .
$$

From this result, the position and width of the peak for small $T$ immediately follow (e.g., for $K^{\mathrm{FL}}=1$ it is easy to see that $\omega_{\max }=\pi T$, and that the width goes as $1 / T$ ). In figure 3 , we compare the values of $\omega_{\max }$ obtained from the numerical spectra with this approximation for $K^{\mathrm{FL}}=0.3$.

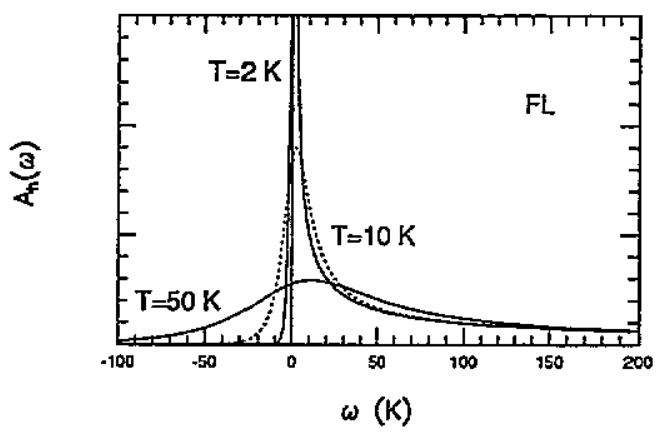

Figure 2. Spectral density for the FL case, with $K^{\mathrm{FL}}=0.3$ and $\omega_{\mathrm{c}}=1500 \mathrm{~K} . A_{\mathrm{b}}(\omega)$ has arbitrary units.

Thus, for an FL, when $T$ decreases, the asymmetric peak becomes narrower, and its position shifts towards zero frequency, while its height increases. The large- $\omega$ behaviour always remains a power law [21]. For a more detailed discussion of the temperature dependence of the $\mathrm{X}$-ray edge effect in an $\mathrm{FL}$, we refer to Ohtaka and Tanabe [15].

We now turn to the spectral density in the case of an MFL. In figures 4 and 5 , we plot the spectral density for this case for two values of $K^{\mathrm{MFL}}, K^{\mathrm{MFL}}=15$ and $K^{\mathrm{MFL}}=400$ (in K). These figures illustrate that the core hole spectra do indeed behave quite differently from that of the FL case: the broadening of the profile with temperature is quite weak; instead, the most salient feature is the shift of the profile 


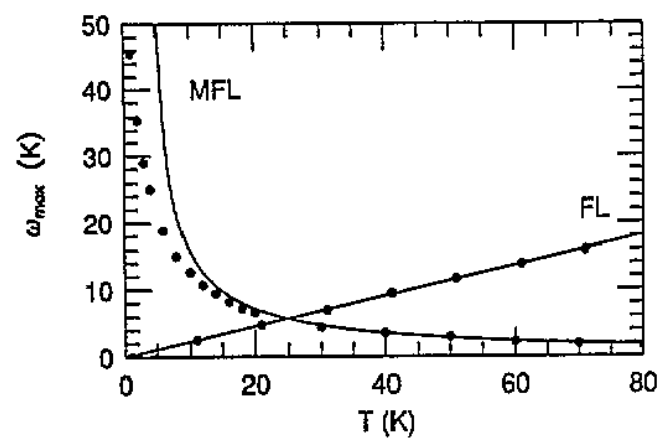

Figure 3. Behaviour of the location of the peak as a function of temperature, with $K^{\mathrm{MFL}}=15 \mathrm{~K}, K^{\mathrm{FL}}=0.3$ and $\omega_{c}=1500 \mathrm{~K}$. The points are from the numerical spectra; the lines are from the approximations discussed in the text.

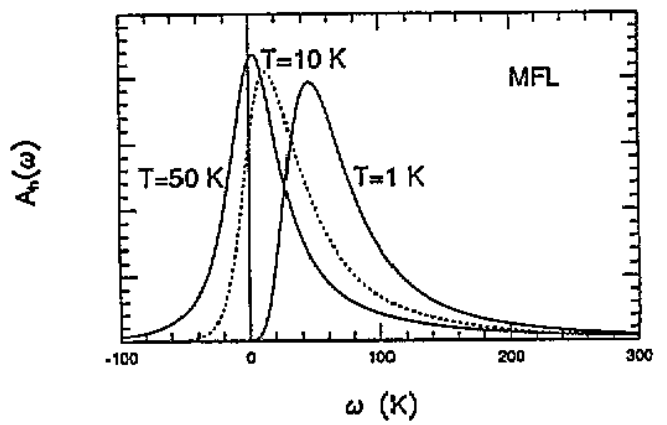

Figure 4. Spectral density in arbitrary units for the MFL case, $K^{\mathrm{MFL}}=15 \mathrm{~K}$ and $\omega_{c}=1500 \mathrm{~K}$.

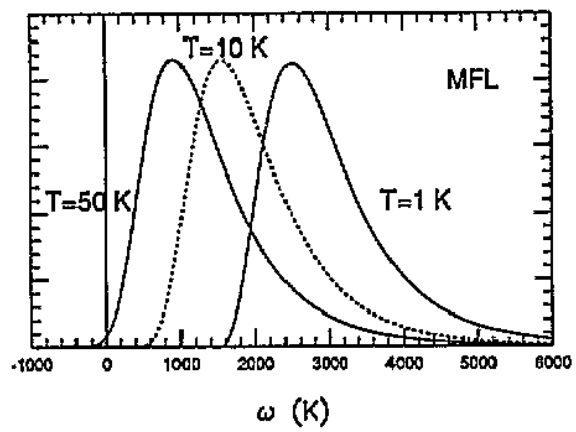

Figure 5. Spectral density in arbitrary units for the MFL case, $K^{\mathrm{MFL}}=400 \mathrm{~K}$ and $\omega_{\mathrm{c}}=1500 \mathrm{~K}$.

position with temperature. This effect is stronger the larger $K^{\mathrm{MFL}}$ is, and is due to the fact that the temperature dependence of $\operatorname{Re} F_{2}^{\mathrm{MFL}}$ and $\operatorname{Im} F_{2}^{\mathrm{MFL}}$ is reversed relative to that of an ordinary $\mathrm{FL}$.

Along similar lines as above, one can derive an approximate expression for the 
peak region of $A_{\mathrm{h}}(\omega)$ from the $t \rightarrow \infty$ asymptotic behaviour of $F_{2}^{\mathrm{MFL}}$ derived in the previous section. The result is

$$
A_{\mathrm{h}}^{\mathrm{MFL}}(\omega) \approx \frac{K^{\mathrm{MFL}} \pi \cos \left(K^{\mathrm{MFL}} \pi / 4 T\right)+2 \omega \sin \left(K^{\mathrm{MFL}} \pi / 4 T\right)}{\left(K^{\mathrm{MFL}} \pi\right)^{2} / 4+\omega^{2}} .
$$

This expression is only accurate for small $K^{\mathrm{MFL}}$ and large $T$ : the first condition is necessary to ensure that $|\rho(t)|$ is not too small in the large-time asymptotic regime, and the second condition is necessary to ensure that $\operatorname{Im} F_{2}^{\mathrm{MFL}}$ reaches its asymptotic behaviour relatively quickly, compare figure 1 .

In figure 3 we also show data of $\omega_{\max }$ for the MFL spectra obtained numerically, with $K^{\mathrm{MFL}}=15 \mathrm{~K}$. The solid line is the result obtained for $\omega_{\max }$ from equation (17); as expected, this approximation is accurate for large $T$, but breaks down at small $T$.

The following approximation helps to understand the physics underlying the above behaviour of $A_{\mathrm{h}}^{\mathrm{MFL}}(\omega)$. In the limit of small $T, \operatorname{Im} F_{2}^{\mathrm{MFL}}$ crosses over so slowly to its asymptotic behaviour that we can actually use the approximation $\operatorname{Im} F_{2}^{\mathrm{MFL}}(t) \approx \mathrm{d} \operatorname{Im} F_{2}^{\mathrm{MFL}}(t) /\left.\mathrm{d} t\right|_{0} t$ from equation (15) throughout most of the the regime where $\operatorname{Re} F_{2}^{\mathrm{MFL}}$ has already reached its asymptotic behaviour.

$$
A_{\mathrm{h}}^{\mathrm{MFL}}(\omega) \approx K^{\mathrm{MFL}} \pi /\left\{\left(K^{\mathrm{MFL}} \pi\right)^{2} / 4+\left[\omega-K^{\mathrm{MFL}} \ln \left(1.13 \omega_{\mathrm{c}} / T\right)\right]^{2}\right\} .
$$

This approximation becomes better the larger $K^{\mathrm{MFL}}$ is, provided it is still smaller than $\omega_{c}$, and holds for $\omega$ close to the peak value [22]. According to equation (18), the peak value $\omega_{\max }$ has a logarithmic temperature dependence for small $T$. This due to the fact that the effective mass of an MFL diverges as $\ln T$ as $T \rightarrow 0$ (the data in figure 5 for small $T$ are consistent with this result).

In practice, the detailed spectrum $A_{\mathrm{b}}(\omega)$ cannot be measured in XPS, as a result of the broadening caused by the finite lifetime of the core hole. This broadening can be of the order of an $\mathrm{eV}$. As pointed out by Doniach and Šunjic [10] the $\mathrm{x}$-ray edge effect in an FL gives rise to an asymmetric line shape. In principle, the asymmetry is slightly temperature dependent, but often thermal broadening from phonons gives a bigger temperature dependence [11]. Our results above show that the line shape in an MFL should be less asymmetric, but that there should be a temperature dependent shift of the line as a whole if $K^{\mathrm{MFL}}$ is large enough. Since XPS experiments can be done with meV accuracy, our very crude estimate $K^{\mathrm{MFL}} \approx 1000 \mathrm{~K}(\approx 80 \mathrm{meV})$, together with the result $\omega_{\max } \approx K^{\mathrm{MFL}} \ln \left(1.13 \omega_{\mathrm{c}} / T\right)$ obtained from (18) indicate that this result should be measurable in principle. In the cuprate superconductors, the main experimental challenge may be to find a suitable core level that interacts sufficiently strongly with the conduction electrons in the copper oxide planes.

\section{The hopping rate}

Finally, let us discuss the behaviour at zero frequency of $A_{\mathrm{h}}^{\mathrm{MFL}}(\omega)$, which is proportional to the hopping rate. From the spectra, figures 4 and 5 , it is immediately clear that $A_{\mathrm{b}}^{\mathrm{MFL}}(\omega=0)$ decreases with decreasing temperature, in contrast with the FL case where $A_{\mathrm{h}}^{\mathrm{FL}}(\omega=0)$ goes up with decreasing temperature as can be seen in figure 2. For large temperatures, we can use equation (17) at zero frequency to obtain

$$
\nu^{\mathrm{MFL}}=2 \Delta^{2} \cos \left[K^{\mathrm{MFL}} \pi / 4 T\right]\left(4 / K^{\mathrm{MFL}} \pi\right)
$$


The analogous expression given by Zhang $e t$ al, although not quite right, gives the same qualitative behaviour. Combination with equation (18) gives

$$
\nu^{\mathrm{MFL}}(T \rightarrow 0) / \nu^{\mathrm{MFL}}(T=\infty)=1 /\left(1+\left(4 / \pi^{2}\right) \ln ^{2}\left(1.13 \omega_{\mathrm{c}} / T\right)\right) \quad(\text { low } T)
$$

independent of $K^{\mathrm{MFL}}$. The logarithmic vanishing of the hopping rate as $T \rightarrow 0$ is again associated with the logarithmic divergence of the effective mass as $T \rightarrow \infty$ in the MFL model. At large and intermediate temperatures, the rate of decrease of the hopping rate $\nu^{\mathrm{MFL}}$ with decreasing temperature does depend on $K^{\mathrm{MFL}}$, the suppression being larger the larger $K^{\mathrm{MFL}}$ is. This is illustrated in figure 6 for two relatively small values of $K^{\mathrm{MFL}}$. Since quantum effects of the type considered here can only be studied experimentally at rather low temperatures (less than $50 \mathrm{~K}$, say), we see that in order to observe the sharp decrease in the hopping rate as a function of temperature, $K^{\mathrm{MFL}}$ should not be too large (of course, it also requires a material that exhibits MFL behaviour down to very low temperatures).

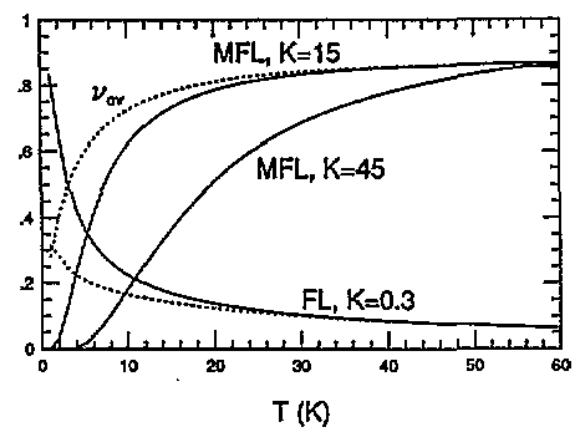

Figure 6. Hopping rate as a function of temperature, relative to the high-temperature value.

The above analysis corresponds to the ideal case. It is well known that if there are impurities, there is a distribution of levels, and one has to average $A(\omega)$ over a region around zero frequency to obtain the hopping rate [12] (this may be particularly relevant for the high- $T_{c}$ materials). As figure 2 shows, in the FL case, there is a frequency range in the spectrum where the values for a low temperature are higher than at a high temperature, but further away from zero frequency it is the other way around. Averaging therefore leads to a flattening of the curve for the hopping rate, especially at low temperatures, where the peaks are narrow. In the MFL case, we have seen in figure 4 that the value in zero decreases upon lowering the temperature, but also that for intermediate temperatures the peak remains nearby and that its height remains roughly the same. Averaging over frequencies near zero therefore leads to a flattening at larger temperatures and a shift of the crossover region to smaller temperatures. The difference between $\mathrm{FL}$ and $\mathrm{MFL}$ is thus less pronounced than in the ideal case. In figure 6 , the dotted curves are the result of averaging our numerically obtained spectra over a region $\Delta \omega=10 \mathrm{~K}$. 


\section{Conclusion}

In this paper we have compared the difference between the effects of interaction with an FL and an MFL on the hopping rate of a particle and on the XPS spectrum of a deep core hole. As we have seen, in the case of coupling to an MFL, the increasing effective mass of the MFL (or the fact that the quasi-particle spectral weight vanishes as $T \rightarrow 0$ ) causes a decrease of the hopping rate with decreasing temperature, instead of the usual increase. Only when the coupling strength is relatively weak do we expect the characteristic crossover to occur in a temperature regime where in earlier experiments in normal metals the quantum effects have turned out to be measurable. In addition, we expect two other effects to adversely affect the experimental testability of the predicted decrease in the hopping rate in high- $T_{\mathrm{c}}$ superconductors. First of all, it will be difficult to disentangle such a decrease from the usual classical activated behaviour $\left(\propto \mathrm{e}^{-E / k T}\right.$ ) of the diffusion coefficient. Moreover, in high- $T_{c}$ superconductors with their complicated unit cell, the effect might easily be washed out to a large degree by the hopping of the particle between several non-equivalent sites, at some of which the coupling to the conduction electrons in the $\mathrm{CuO}$ planes will be small.

The main feature of the XPS spectrum of a deep core hole interacting with an MFL is found to be a temperature dependent shift of the position of the peak. This effect in principle offers better opportunities for experimental tests. For, in a photoemission experiment the core level of a given atom at a fixed site in the unit cell can be measured. Furthermore, the meV resolution with which the spectra can be measured should allow one to observe this shift provided $K^{\mathrm{MFL}}$ is not much smaller than our rough estimate $K^{\mathrm{MFL}} \approx 75 \mathrm{meV}$ (cf. equation (10)). We hope our findings will stimulate the experimental search for this effect in photoemission experiments.

\section{Acknowledgments}

We are grateful to G A Sawatzky for a stimulating discussion.

\section{References}

[1] Varma C M, Littlewood P B, Schmitt-Rink S, Abrahams E and Ruckenstein A E 1989 Phys. Rev. Lett. 631996

[2] Littlewood P B and Varma C M 1991 J. Appl Phys. 69 4979; 1992 Phys. Rev. B 46405

[3] Zhang C, Toyoda T and Takahashi Y 1992 J. Phys: Condens. Matter 4 L15

[4] Zhang C, Gumbs G and Tzoar N 1991 Phys. Rev. B 431463

[5] Yamada K, Sakurai A and Theshige M 1983 Prog. Theor. Phys. 7073

[6] Kondo $J$ and Yoshimori A (ed) 1988 Fermi Surface Effects (Berlin: Springer)

[7] Anderson P W 1967 Phys. Rev: Leth 181049

[8] Mahan G D 1981 Many-Particle Physics (New York: Plenum)

[9] Almbladh C-O and Minnhagen P 1978 Phys. Status Solidi b 85135

[10] Doniach S and Šunjić M $1970 \mathrm{~J}$. Phys. C: Solid State Phys. 3285

[11] Citrin P H, Wertheim G X and Baer Y 1977 Phys. Rev. B 164256

[12] Sugimoto H 1986 J. Phys. Soc. Japan 551687

[13] Kondo J 1976 Physica B 84 40; 1984 Physica B 123 175; 1984 Physica B 12425

[14] Nozières P and de Dominicis C T 1969 Phys. Rev 1781097

[15] Ohtaka K and Tanabe Y 1990 Rev. Mod. Phys. 62929

[16] Lee D K and Chen Y 1992 Phys. Rev. Lett. 691399

[17] Ogawa T, Furusaki A and Nagaosa N 1992 Phys. Rev. Lett. 683638 
[18] Pelzer F 1991 Phys. Rev. B 44293

[19] These difficulties are less manifest when one confines the analysis to properties associated with the self-energy. For, the strength of the anomalous terms in the self-energy is the product of the prefactor of the polarizability and an interaction parameter. For most properties, only this product is imponant, but not the separate constants. See e.g. [18] for an estimate of the coupling strength from the conductivity data.

[20] This estimate is based on the assumption that $E_{\mathrm{F}} \approx 1 \mathrm{eV}$ as most measurements as well as as a simple estimate using a hopping parameter of about $0.5 \mathrm{eV}$ indicate.

If the much lower estimate $E_{\mathrm{F}} \approx 0.1 \mathrm{eV}$ of Rietveld $\mathrm{G}$, Chen N Y and van der Marel D 1992 Phys. Rev. Lett 692578 is used, one obtains an even larger value of $K^{\mathrm{MFL}}$.

[21] The starting model is, however, only realistic for small values of $\omega$. See e.g. [8].

[22] We note that formally the spectrum $A_{\mathrm{b}}^{\mathrm{MFL}}(\omega)$ calls off as a Gaussian for frequencies $\left|\omega-\omega_{\max }\right|>$ $\omega_{c}$, due to the fact that $\operatorname{Re} F_{2}^{\mathrm{MFL}}$ goes as $t^{2}$ for times $t<\omega_{\mathrm{c}}^{-1}$. Of course, this behaviour is just an artifact of the unrealistic behaviour of $\operatorname{Im} P^{M F L}(\omega)$ for $\omega>\omega_{c}$. 